\title{
Hawk mimicry does not reduce attacks of cuckoos by highly aggressive hosts
}

\author{
Laikun Ma(i), Canchao Yang (10) and Wei Liang ${ }^{*}$ (1)
}

\begin{abstract}
Background: Resemblance to raptors such as hawks (Accipiter spp.) is considered to be an adaptive strategy of cuckoos (Cuculus spp.), which has evolved to protect cuckoos against host attacks. However, the effectiveness of the mimicry remains controversial, and is not yet fully studied for highly aggressive hosts.

Methods: We evaluated the effectiveness of sparrowhawk (Accipiter nisus) mimicry by common cuckoos (Cuculus canorus) in oriental reed warblers (Acrocephaus orientalis), which are highly aggressive hosts. Using a both the single and the paired dummy experiment, defense behaviors and attack intensities of oriental reed warblers against common cuckoos, sparrowhawks and oriental turtle doves (Streptopelia orientalis) were assessed.
\end{abstract}

Results: Oriental reed warblers exhibit strong nest defense behaviors, and such behaviors do not change with breeding stage (i.e., egg stage and nestling stage). Furthermore, assistance from conspecific helpers may increase attack intensities. However, they were deterred from mobbing overall by the presence of the hawk.

Conclusions: Oriental reed warblers are able to distinguish cuckoos from harmless doves. However, they may be deterred from mobbing by the presence of the predatory hawk, suggesting hawk mimicry may be ineffective and does not reduce attacks of cuckoos by highly aggressive hosts.

Keywords: Attack, Brood parasitism, Common cuckoo, Mobbing, Nest defense, Oriental reed warbler

\section{Background}

The confrontation between adaptation and counteradaptation of specific avian brood parasites, such as cuckoos, and their hosts, is a classic example of co-evolution (Davies 2000, 2011; Soler 2014). The high costs of successful cuckoo parasitism have forced hosts to evolve counter-adaptive strategies in different stages. For instance, during the egg laying and incubation stage, many hosts have developed the ability to recognize foreign eggs (Davies and Brooke 1988, 1989; Brooke and Davies 1988; Stokke et al. 2002; Spottiswoode and Stevens 2010, 2011; Yang et al. 2016). During the nestling stage, hosts are able to identify foreign chicks (Langmore et al. 2003, 2009; Grim 2007; Sato et al. 2010; Yang et al. 2015; Huo et al. 2018). Usually, cuckoos take one host egg during parasitism (Chance 1940; Davies 2000), which

*Correspondence: liangwei@hainnu.edu.cn

Ministry of Education Key Laboratory for Ecology of Tropical Islands,

College of Life Sciences, Hainan Normal University, Haikou 571158, China means that, once cuckoo eggs are laid, hosts suffer various levels of reproductive losses, even if they perform successful defense behaviors afterwards. Therefore, preventing cuckoos from laying eggs, i.e. nest defense, is the most effective anti-parasitism strategy (Welbergen and Davies 2009; Feeney et al. 2012).

The competition between hosts and brood parasites during the egg laying and incubation stage has received much attention. Many hosts demonstrate strong aggressive nest protection behaviors against cuckoos approaching the nests, to prevent parasitism (Welbergen and Davies 2009). This leads to huge costs for the cuckoos (Davies and Brooke 1988; Welbergen and Davies 2008), and may even result in the death of the cuckoos (Molnár 1944). In addition, hosts' nest defenses against cuckoos not only prevent cuckoo parasitism (Welbergen and Davies 2009), but such defense behaviors can be passed on to other individuals through learning, hence strengthening anti-parasitism of the population (Davies 
and Welbergen 2009; Thorogood and Davies 2012, 2016; Feeney and Langmore 2013).

Successful nest defense forces cuckoos to develop more effective parasitism strategies, and mimicking hawks (Accipiter spp.) is thought to be an adaption of cuckoos against host attacks (Langmore and Kilner 2010; Welbergen and Davies 2011; Feeney et al. 2012; Thorogood and Davies 2013a, b). Hawks are typical small birds of prey, and pose more direct threats to adult birds (Götmark and Post 1996). Resemblance to hawks enables cuckoos to confuse and alarm hosts, and reduce attacks from hosts (Davies and Welbergen 2008; Welbergen and Davies 2011). A recent study discovered that calls of female common cuckoos mimic those of sparrowhawks to some extent, which resulted in similar intimidation (York and Davies 2017). However, the effectiveness of cuckoos' hawk mimicry has only been demonstrated for a few host species, among which only reed warblers (Acrocephalus scirpaceus) are main hosts of common cuckoos (Cuculus canorus) (Davies and Welbergen 2008; Langmore and Kilner 2010; Welbergen and Davies 2011; Thorogood and Davies 2013b; Liang 2017; York and Davies 2017). Reed warblers lack aggressiveness, and are not strong nest defenders, although nest defense levels in reed warblers vary significantly between different species (Montgomerie and Weatherhead 1988). Therefore, results from studies on reed warblers cannot be extrapolated to other hosts. In fact, many hosts can accurately identify the differences between cuckoos and sparrowhawks, and respond with different defense levels (Duckworth 1991; Grim 2005; Welbergen and Davies 2008; Campobello and Sealy 2010; Feeney et al. 2012; Trnka and Prokop 2012; Li et al. 2015; Liang and Møller 2015; Yu et al. 2016, 2017). For example, Smith and Hosking (1955) discovered that many small passerines (not the main hosts of cuckoos) fear sparrowhawk dummies, but attack cuckoo dummies aggressively, suggesting their abilities to distinguish the two. Paired experiments with great reed warblers (Acrocephalus arundinaceus) by Trnka and Prokop (2012) revealed that, when presented simultaneously with paired dummies (of common cuckoo, sparrowhawk Accipiter nisus, and turtle dove Streptopelia turtur), great reed warblers exhibit aggressive attack behaviors to both dummies, with higher attack intensities towards cuckoos and sparrowhawks. However, a study by Feeney et al. (2015) suggested that tawny-flanked prinia (Prinia subflava) hosts were equally aggressive towards female cuckoo finches (Anomalospiza imberbis) and southern red bishops (Euplectes orix), suggesting successful mimicry of female bishops by female cuckoo finches.

The oriental reed warbler (Acrocephaus orientalis) is one of the main hosts of common cuckoos, and the two have reached a relatively high "arms race level" after long-term coevolution (Yang et al. 2014, 2016, 2017; Li et al. 2016). Oriental reed warblers are highly aggressive towards intruders, and can adjust their attack intensities according to the level of threat and familiarity of the intruders ( $\mathrm{Li}$ et al. 2015, 2016) or under variable parasitism pressures (Lindholm and Thomas 2000). In great reed warblers, they attacked cuckoos and sparrowhawks more often than turtle doves. However, when confronted with the simultaneously presented mounts, they attacked the cuckoo more frequently than the sparrowhawk (Trnka and Prokop 2012). In addition, Trnka and Prokop (2012) found that attack behaviors of great reed warblers towards common cuckoos weaken with nest stage (but see Moskát 2005).

In this study, we tested a different population of oriental reed warblers during the egg laying, early incubation and nestling stages of nest cycle. Oriental reed warblers were presented with three different types of single dummies, namely common cuckoos, sparrowhawks and oriental turtle doves (Streptopelia orientalis), as well as paired dummies prepared by combining different dummies. By conducting these experiments, we aimed to evaluate the ability of oriental reed warblers to correctly identify potential brood parasites, and to assess nest defense behaviors towards different nest intruders.

\section{Methods}

\section{Study area and study species}

The study area is located in the Yongnianwa wetland in Yongnian County, Hebei Province, China $\left(36^{\circ} 40^{\prime} 60^{\prime \prime}-36^{\circ} 41^{\prime} 06^{\prime \prime} \mathrm{N}, \quad 114^{\circ} 41^{\prime} 15^{\prime \prime}-114^{\circ} 45^{\prime} 00^{\prime \prime} \mathrm{E}\right)$. The Yongnianwa wetland is a natural depression in the alluvial plain of the Fuyang River, a tributary of the Hai River. It is located at the intersection of the Fuyang River and the Zhang River. The area is in a temperate semi-humid continental monsoon climate zone and includes mature hydrological systems with many river branches. The wetland is located at only $40.3 \mathrm{~m}$ above sea level, and is filled with water all year round. The average annual rainfall is $527.8 \mathrm{~mm}$ which concentrated in the summer and the annual average temperature is $12.9^{\circ} \mathrm{C}$. The main plant species in the wetland are reed (Phragmites australis) mixed with cattail (Typha latifolia), and other herbaceous plants (Ma et al. 2018). A total of 257 oriental reed warbler nests with an overall parasitism rate of $14.8 \%$ were recorded during the breeding seasons 2016-2017 (Ma et al. 2018).

\section{Field data collection}

Field work was carried out during the bird breeding seasons (April-August) of 2016 and 2017. Breeding nests of oriental reed warblers were systematically searched within the study area, and the breeding stage 
of each nest was determined. Oriental reed warbler nest defense behaviors were investigated using dummies (stuffed birds) of common cuckoos $(27.7 \mathrm{~cm}$ in body length), sparrowhawks (33.2 $\mathrm{cm}$ in body length), and oriental turtle doves (25.8 cm in body length). Common cuckoos are common brood parasites in the study area. Sparrowhawks are typical predators of passerine birds and are common summer breeding hawks in northern China (Zheng 2017). Oriental turtle doves are commonly seen breeding birds in the study area. Two dummies were taken from each bird species and one of them was randomly selected in the experiments in order to avoid pseudo-replication. Hosts' responses to the dummies included the following scale, from low to high levels of aggressiveness: (1) no response, the bird was not observed or watched the mount silently without clear responsive behavior; (2) alert, the bird approached the nest (hopped onto the reed stems), but gave alert calls from a safe distance (more than $1 \mathrm{~m}$ ); (3) mobbing, the bird jumped or flew around/over the dummy, persistently giving alarm and distress calls; and (4) attack, the birds physically attacked the dummy. The first two responses are collectively considered to be non-attack behaviors, and the latter two are collectively considered to be attack behaviors (also see Li et al. 2015). The responsive behavior of the bird was recorded by L.M. hidden approximately 4-5 $\mathrm{m}$ away from the nest site and other variables like number of attacks were extracted from subsequent analyses of video recordings in the laboratory. The minicamera (size: $57 \mathrm{~mm} \times 20 \mathrm{~mm} \times 18 \mathrm{~mm}$ ) was fixed at a distance of approximately $2 \mathrm{~m}$ in front of the nest and dummy.

\section{Single dummy experiment}

All single dummy experiments were completed during the 2016 breeding season. One dummy was randomly selected before an experiment. It was fixed at a distance of $0.5 \mathrm{~m}$ in front of an oriental reed warbler nest with a stand, and was kept standing with its head facing the nest. The 5-min responses of oriental reed warblers to the dummy were observed and recorded when the bird appeared around the dummy (also see Li et al. 2015). Subsequently, the next experiment was carried out at least $1 \mathrm{~h}$ later to minimize the effects of carry-over aggression (Gill et al. 2008). Each nest was presented with three mounts in random order and the sequence was completed within 1-2 days.

\section{Paired dummy experiment}

The three types of dummies were combined in pairs as follows: common cuckoo versus turtle dove (Experiment 1), common cuckoo versus sparrowhawk (Experiment 2) and sparrowhawk versus turtle dove (Experiment 3). One pair of dummies was randomly selected before an experiment, and one direction of an oriental reed warbler nest was randomly chosen. The two dummies, placed $0.8 \mathrm{~m}$ away from each other, were fixed at a distance of $0.5 \mathrm{~m}$ in front of the nest with two stands, and were kept standing with their heads facing the nest (also see Trnka et al. 2012). The responses of oriental reed warblers to the dummies were observed. Each experiment began at the moment of the first contact-attack of one bird and lasted $1 \mathrm{~min}$ when the mounts were immediately removed to prevent them from being damaged. The attack intensity towards each of the two dummies was expressed in relation to the absolute number of attacks within 1 min since the first attack which was extracted from subsequent analyses of video recordings in the laboratory. Only one experiment was performed for each nest either in egg stage or nestling stage.

\section{Data analysis}

Statistical analyses were performed using SPSS 16.0. For the single dummy experiment, generalized linear mixed models (GLMM) were used to analyze the effect of dummy types and nest stage on defense behaviors of oriental reed warblers. For the paired dummy experiment, GLMMs were used to analyze the effects of nest stage on the defense levels of oriental reed warblers. Wilcoxon signed ranks test was used to investigate variations in defense levels in response to different dummies in each paired experiment. The significance level was set at $p=0.05$, and variables were considered to be highly significant when $p<0.01$.

\section{Results}

\section{Single dummy experiments}

In total, 39 dummy experiments were performed (Fig. 1), including two sets of single dummy experiments for

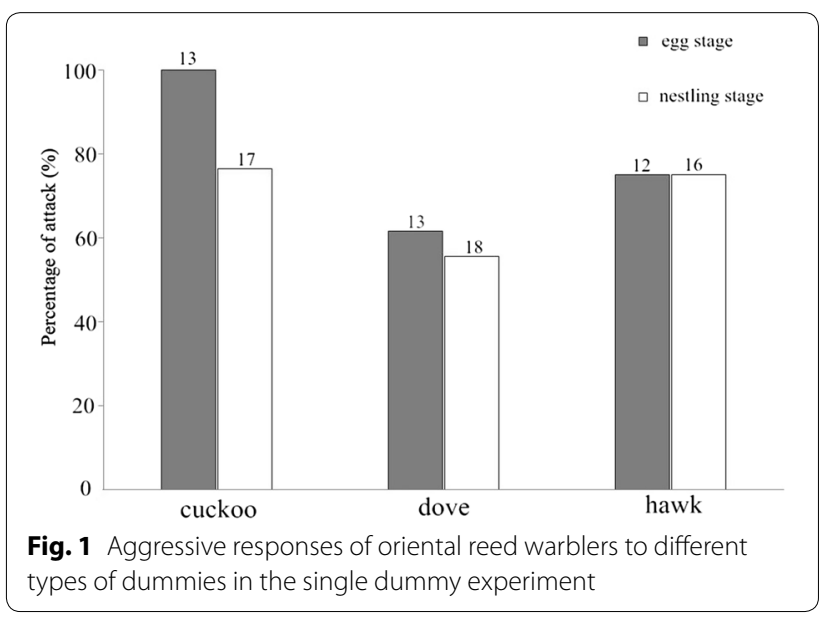


four nests which one nest of egg stage was lack of hawk dummy experiment and three nests of nestling stage were lack of two hawk dummy experiments and one cuckoo dummy experiment, and three sets of single dummy experiments for the remaining nests. Thus, single dummy experiments were performed for 31 nests of oriental reed warblers, with 13 nests in the egg incubation stage, and 18 nests in the nestling stage.

All oriental reed warblers performed for single dummy experiments showed strong nest defense behaviors, and $90 \%(28 / 31)$ of the oriental reed warblers attacked the dummies. Attacks were observed in $73 \%$ of all experiments $(65 / 89)$, and $69 \%$ of the attacks involved neighbor participation (45/65), with the number of helpers ranging from 1 to 11. Nest defense behaviors were not affected by dummy type nor nest stage (dummy type: $F=2.351$, $p=0.102$; nest stage: $F=0.000, p=1.000$ ).

\section{Paired dummy experiments}

In total, 63 nests of oriental reed warblers were tested for paired dummy experiments either in egg stage or in nestling stage. The experiment 1 was performed for 7 nests in the egg stage and 12 nests in the nestling stage. The experiment 2 was performed for 11 nests in the egg stage and 11 nests in the nestling stage. The experiment 3 was performed for 10 nests in the egg stage and 11 nests in the nestling stage.

Attack intensities of oriental reed warblers towards paired dummies did not vary with nest stage (cuckoo vs. dove: $F=0.069, p=0.794$; cuckoo vs. hawk: $F=0.061$, $p=0.807$; hawk vs. dove: $F=0.118, p=0.735$ ). Therefore, all data from the egg incubation and nestling stages were combined for analysis. There was a significant difference in the number of attacks directed to each dummy in the dummy combination of common cuckoo versus turtle dove, and the number of attacks directed at common cuckoos was significantly higher than that to turtle doves (Wilcoxon signed ranks test, $Z=-3.529$, $p<0.001)$. However, we found no significant attack preferences for the other dummy pairs (Fig. 2; cuckoo vs. hawk: $Z=-1.038, p=0.299$; hawk vs. dove: $Z=-0.035$, $p=0.972)$.

\section{Discussion}

The main findings of this study were that oriental reed warblers displayed aggressive nest defense behaviors with more than $90 \%$ of the warblers demonstrating strong attack behaviors towards intruders, and their nest defense behaviors did not differ between egg laying, incubation, and nestling stages. Our results were similar to previous work on reed warblers, which found few differences in overall attacks at different stages (Li et al. 2015), although rasp call rate towards cuckoos declined significantly

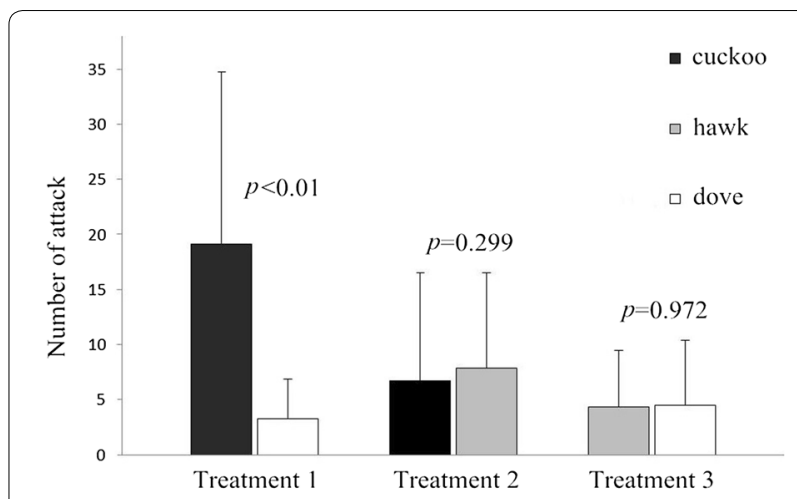

Fig. 2 Intensity of aggressive responses of oriental reed warblers to different types of dummies in the paired dummy experiment

between egg laying and chick rearing stages (Campobello and Sealy 2010). In addition, long time interval among different dummy experiments would minimize the effects of carry-over aggression.

However, the results on the attack intensities of oriental reed warblers towards different types of attackers are not in agreement with the results found in another population of oriental reed warblers (Li et al. 2015) and great reed warblers (Trnka et al. 2012). In our study, oriental reed warblers were able to distinguish common cuckoos from harmless turtle doves, and tended to be more likely to mob cuckoos than sparrowhawks, although this tendency was not significant perhaps due to the low sample size for each presentation. These results imply that oriental reed warblers are highly aggressive towards any model placed at their nests regardless of mimicry.

Many hosts are known to violently expel and attack cuckoos near their nests, causing different levels of damage to cuckoos (Wyllie 1981; Davies and Brooke 1988; Welbergen and Davies 2008), and even death (Molnár 1944). This reduces the chances of successful cuckoo parasitism (Welbergen and Davies 2009; Feeney et al. 2012; Thorogood and Davies 2016). The strong attacks of oriental reed warblers towards cuckoo dummies in our study resemble those of other hosts. Meanwhile, in paired experiments, when facing familiar intruders, namely common cuckoos and turtle doves, simultaneously, oriental reed warblers demonstrated significant selective aggression towards common cuckoos, indicating their abilities to distinguish the two intruders. This is in accordance with previous research on oriental reed warblers (Li et al. 2015).

However, in both single and paired dummy experiments, the responses of oriental reed warblers to sparrowhawks were not significantly different from their responses to common cuckoos $(Z=-1.038, p=0.299)$ and doves $(Z=-0.035, p=0.972)$. In particular, in the 
paired dummy experiments, cuckoos were attacked much less often when they were presented alongside a hawk than a dove. This was different from the behaviors displayed by oriental reed warblers in the population studied by Li et al. (2015), as well as the great reed warbler populations of European lineage (Moskát 2005; Trnka and Prokop 2012; Trnka et al. 2012), suggesting that oriental reed warblers in our population may be deterred from mobbing overall by the presence of the predatory hawk. Interestingly, Li et al. (2015) also found that the magpie (Pica pica), a commonly seen but larger nest/egg predator, were attacked much less often by oriental reed warblers.

In reed warblers, hosts' mobbing rates varied depending on cuckoo parasitism risk (Thorogood and Davies 2013a), and also by the risk of encountering a hawk if mistaking it for a cuckoo (Thorogood and Davies 2012, $2013 b)$. In our study population of oriental reed warblers, the cuckoo parasitism rate was $14.8 \%$ (Ma et al. 2018), which was lower than that of other populations in China (27.3\%; Yang et al. 2014), great reed warblers (64\%; Moskát and Honza 2002) and reed warblers (31\%; Trnka and Prokop 2011) in Europe. Such low parasitism might explain why oriental reed warblers displayed aggressive nest defense behaviors towards cuckoos suggesting that hawk mimicry may be ineffective in our population of oriental reed warblers.

Another possibility was that oriental reed warblers are aggressive in general, and tend to expel any intruders. Our results indicated that even for familiar yet harmless doves, oriental reed warblers exhibited an attack rate as high as $58 \%(18 / 31)$, and even attacked people who approached their nests (L.M., personal observations). Among all attack behaviors, helper-assisted attacks occur in up to $69 \%$ of the nests studied. We suggest that the presence of helpers may reinforce the oriental reed warblers' desires to attack, and make them much bolder when facing intruders. In a study on European pied flycatchers (Ficedula hypoleuca), the existence of helpers was found to enhance European pied flycatchers' aggression and their desire to attack predators, and birds may sense the presence of helpers when attacking intruders (Krams et al. 2009, 2012). In our study, the presence of helpers within the population is much higher than in the population in other studies ( $\mathrm{Li}$ et al. 2015, 2016), which may be due to different population densities and parasitism rates in different areas. Nonetheless, further studies are needed to investigate whether this may lead to variations in nest defense behaviors.

In a European study on great reed warblers, it was discovered that their attack behaviors towards common cuckoos weaken with nest stage (Trnka and Prokop 2012). This is because, although common cuckoos may visit host nests at any stage, most eggs are laid during the egg laying stage, and parasitism pressure is reduced during the nestling stage (Honza et al. 2002). Other studies, however, suggested that great reed warblers' nest defense did not weaken with the advancement of nest stage, and that the attack intensities remained relatively high during the nestling stage (Moskát 2005). In our study, no changes in nest defense levels with the nest stages were found for oriental reed warblers, which agreed with previous work (Campobello and Sealy 2010; Li et al. 2015). Relative to the less aggressive hosts, the high aggressiveness of oriental reed warblers may be a broad nest defense behavior and thus does not vary with nest stage and intruder types. Another opinion is that cuckoos are not only nest parasites, but also nest predators, which has been reported for a number of host species (Davies and Brooke 1988; Moksnes et al. 2000; Briskie 2007; Su et al. 2017).

\section{Conclusions}

In this study, we showed that oriental reed warblers not only exhibit strong nest defense behaviors, but that they also employ a variety of defenses intended to expel and attack any intruders within their nest-sites. Furthermore, assistance from conspecific helpers may increase oriental reed warblers' attack intensities. In addition, nest defense behaviors in oriental reed warblers did not change with breeding stage (i.e., egg stage, nestling stage). Our study indicated that oriental reed warblers are able to distinguish cuckoos from harmless doves. However, they may be deterred from mobbing by the presence of the predatory hawk, suggesting hawk mimicry may be ineffective and does not reduce attacks of cuckoos by highly aggressive hosts.

\section{Authors' contributions}

WL conceived and designed the experiments. LM conducted the field work. CY and LM performed the data analysis. LM wrote the early draft and WL revised the manuscript. All authors read and approved the final manuscript.

\section{Acknowledgements \\ We would like to thank Rose Thorogood and the anonymous reviewers for helpful comments on an early version of this manuscript. We thank the Forestry Bureau of Yongnian County, Hebei Province, China, for permission to undertake this study including all experimental procedures. We are grateful to Jianping Liu, Jianwei Zhang, Bo Zhou and Xiaodong Rao for their assistance with fieldwork.}

\section{Competing interests}

The authors declare that they have no competing interests. The funders had no role in study design, data collection and analysis, decision to publish, or preparation of the manuscript.

Consent for publication Not applicable. 


\section{Ethical standard}

The experiments comply with the current laws of China. Experimental procedures were in agreement with the Animal Research Ethics Committee of Hainan Provincial Education Centre for Ecology and Environment, Hainan Normal University (Permit No. HNECEE-2012-003).

\section{Funding}

This work was supported by the National Natural Science Foundation of China (Nos. 31672303 to CY, 31472013 and 31772453 to WL).

\section{Received: 31 May 2018 Accepted: 15 October 2018}

\section{Published online: 31 October 2018}

\section{References}

Briskie JV. Direct observations of shining cuckoos (Chrysococcyx lucidus) parasitising and depredating grey warbler (Gerygone igata) nests. Notornis. 2007;54:15-9.

Brooke MdL, Davies NB. Egg mimicry by cuckoos Cuculus canorus in relation to discrimination by hosts. Nature. 1988;335:630-2.

Campobello D, Sealy SG. Enemy recognition of reed warblers (Acrocephalus scirpaceus): threats and reproductive value act independently in nest defence modulation. Ethology. 2010;116:498-508.

Chance EP. The truth about the cuckoo. London: Country Life; 1940.

Davies NB. Cuckoos, cowbirds and other cheats. London: T \& AD Poyser; 2000

Davies NB. Cuckoo adaptations: trickery and tuning. J Zool. 2011;284:1-14.

Davies NB, Brooke MdL. Cuckoos versus reed warblers: adaptations and counteradaptations. Anim Behav. 1988;36:262-84

Davies NB, Brooke MdL. An experimental study of co-evolution between the cuckoo, Cuculus canorus, and its hosts I: host egg discrimination. J Anim Ecol. 1989;58:207-24.

Davies NB, Welbergen JA. Cuckoo-hawk mimicry? An experimental test. Proc $R$ Soc Lond B. 2008;275:1817-22.

Davies NB, Welbergen JA. Social transmission of a host defense against cuckoo parasitism. Science. 2009;324:1318-20.

Duckworth JW. Responses of breeding reed warblers Acrocephalus scirpaceus to mounts of sparrowhawk Accipiter nisus, cuckoo Cuculus canorus and jay Garrulus glandarius. IBIS. 1991;133:68-74.

Feeney WE, Langmore NE. Social learning of a brood parasite by its host. Biol Lett. 2013;9:20130443

Feeney WE, Welbergen JA, Langmore NE. The frontline of avian brood parasite-host coevolution. Anim Behav. 2012;84:3-12.

Feeney WE, Troscianko J, Langmore NE, Spottiswoode CN. Evidence for aggressive mimicry in an adult brood parasitic bird, and generalized defences in its host. Proc R Soc Lond B. 2015:282:20150795.

Gill SA, Neudorf DLH, Sealy SG. Do hosts discriminate between sexually dichromatic male and female brown-headed cowbirds? Ethology. 2008;114:548-56

Götmark F, Post P. Prey selection by sparrowhawks, Accipiter nisus: relative predation risk for breeding passerine birds in relation to their size, ecology and behaviour. Phil Trans R Soc Lond B. 1996;351:1559-77.

Grim T. Host recognition of brood parasites: implications for methodology in studies of enemy recognition. Auk. 2005;122:530-43.

Grim T. Experimental evidence for chick discrimination without recognition in a brood parasite host. Proc R Soc Lond B. 2007;2007(274):373-81.

Honza M, Taborsky B, Taborsky M, Teuschl Y, Vogl W, Moksnes A, Røskaft E. Behaviour of female common cuckoos, Cuculus canorus, in the vicinity of host nests before and during egg laying: a radio telemetry study. Anim Behav. 2002;64:861-8.

Huo J, Yang C, Su T, Liang W, Møller AP. Russet sparrows spot alien chicks from their nests. Avian Res. 2018;9:12.

Krams I, Bērzinšs A, Krama T. Group effect in nest defence behaviour of breeding pied flycatchers, Ficedula hypoleuca. Anim Behav. 2009;77:513-7.

Krama T, Bērziņš A, Rytkönen S, Rantala MJ, Wheatcroft D, Krams I. Linking antipredator behaviour and habitat quality: group effect in nest defence of a passerine bird. Acta Ethol. 2012;15:127-34

Langmore NE, Kilner RM. The coevolutionary arms race between horsfield's bronze-cuckoos and superb fairy-wrens. Emu. 2010;110:32-8.
Langmore NE, Hunt S, Kilner RM. Escalation of a coevolutionary arms race through host rejection of brood parasitic young. Nature. 2003:422:157-60.

Langmore NE, Cockburn A, Russell AF, Kilner RM. Flexible cuckoo chick-rejection rules in the superb fairy-wren. Behav Ecol. 2009;20:978-84.

Li D, Wei H, Zhang Z, Liang W, Stokke BG. Oriental reed warbler (Acrocephalus orientalis) nest defence behaviour towards brood parasites and nest predators. Behaviour. 2015;152:1601-21.

Li D, Zhang Z, Grim T, Liang W, Stokke BG. Explaining variation in brood parasitism rates between potential host species with similar habitat requirements. Evol Ecol. 2016;30:905-23.

Liang W. Crafty cuckoo calls. Nat Ecol Evol. 2017;1:1427-8.

Liang W, Møller AP. Hawk mimicry in cuckoos and anti-parasitic aggressive behavior of barn swallows in Denmark and China. J Avian Biol. 2015;46:216-23.

Lindholm AK, Thomas RJ. Differences between populations of reed warblers in defences against brood parasitism. Behaviour. 2000;137:25-42.

Ma L, Yang C, Liu J, Zhang J, Liang W, Møller AP. Costs of breeding far away from neighbors: isolated host nests are more vulnerable to cuckoo parasitism. Behav Proc. 2018. https://doi.org/10.1016/j.beproc.2018.07.017.

Moksnes A, Røskaft E, Hagen LG, Honza M, Mørk C, Olsen PH. Common cuckoo Cuculus canorus and host behaviour at reed warbler Acrocephalus scirpaceus nests. IBIS. 2000;142:247-58.

Molnár B. The cuckoo in the Hungarian plain. Aquila. 1944;51:100-12.

Montgomerie RD, Weatherhead PJ. Risks and rewards of nest defence by parent birds. Q Rev Biol. 1988;63:167-87.

Moskát C. Common cuckoo parasitism in Europe: behavioural adaptations, arms race and the role of metapopulations. Ornithol Sci. 2005;4:3-15.

Moskát C, Honza M. European cuckoo Cuculus canorus parasitism and host's rejection behaviour in a heavily parasitized great reed warbler Acrocephalus arundinaceus population. IBIS. 2002;144:614-22.

Smith S, Hosking E. Birds fighting: experimental studies of the aggressive displays of some birds. London: Faber and Faber; 1955.

Sato NJ, Tokue K, Noske RA, Mikami OK, Ueda K. Evicting cuckoo nestlings from the nest: a new anti-parasitism behaviour. Biol Lett. 2010;6:67-9.

Soler M. Long-term coevolution between avian brood parasites and their hosts. Biol Rev. 2014;89:688-704.

Spottiswoode CN, Stevens M. Visual modeling shows that avian host parents use multiple visual cues in rejecting parasitic eggs. Proc Natl Acad Sci USA. 2010;107:8672-6.

Spottiswoode CN, Stevens M. How to evade a coevolving brood parasite: egg discrimination versus egg variability as host defences. Proc R Soc Lond B. 2011;278:3566-73.

Stokke BG, Moksnes A, Røskaft E. Obligate brood parasites as selective agents for evolution of egg appearance in passerine birds. Evolution. 2002;56:199-205.

Su T, Shao L, Huo J, Yang C, Liang W. Himalayan cuckoo fed on eggs of Bianchi's warbler. Chin J Ecol. 2017;36:89-93 (in Chinese).

Thorogood R, Davies NB. Cuckoos combat socially transmitted defenses of reed warbler hosts with a plumage polymorphism. Science. 2012;337:578-80

Thorogood R, Davies NB. Reed warbler hosts fine-tune their defenses to track three decades of cuckoo decline. Evolution. 2013a;67:3545-55.

Thorogood R, Davies NB. Hawk mimicry and the evolution of polymorphic cuckoos. Chin Birds. 2013b;4:39-50.

Thorogood R, Davies NB. Combining personal with social information facilitates host defences and explains why cuckoos should be secretive. Sci Rep. 2016;6:19872

Trnka A, Prokop P. Polygynous great reed warblers Acrocephalus arundinaceus suffer more cuckoo Cuculus canorus parasitism than monogamous pairs. J Avian Biol. 2011;42:192-5.

Trnka A, Prokop P. The effectiveness of hawk mimicry in protecting cuckoos from aggressive hosts. Anim Behav. 2012;83:263-8.

Trnka A, Prokop P, Grim T. Uncovering dangerous cheats: how do avian hosts recognize adult brood parasites? PLoS ONE. 2012;7:e37445.

Welbergen JA, Davies NB. Reed warblers discriminate cuckoos from sparrowhawks with graded alarm signals that attract mates and neighbours. Anim Behav. 2008;76:811-22.

Welbergen JA, Davies NB. Strategic variation in mobbing as a front line of defense against brood parasitism. Curr Biol. 2009;19:235-40. 
Welbergen JA, Davies NB. A parasite in wolf's clothing: hawk mimicry reduces mobbing of cuckoos by hosts. Behav Ecol. 2011;22:574-9. Wyllie I. The cuckoo. London: Batsford; 1981

Yang C, Li D, Wang L, Liang G, Zhang Z, Liang W. Geographic variation in parasitism rates of two sympatric cuckoo hosts in China. Zool Res. 2014;35:67-71.

Yang C, Wang L, Chen M, Liang W, Møller AP. Nestling recognition in redrumped and barn swallows. Behav Ecol Sociobiol. 2015;69:1-6.

Yang C, Wang L, Liang W, Møller AP. Egg recognition as antiparasitism defence in hosts does not select for laying of matching eggs in parasitic cuckoos. Anim Behav. 2016;122:177-81.

Yang C, Wang L, Liang W, Møller AP. How cuckoos find and choose host nests for parasitism. Behav Ecol. 2017;28:859-65.
York JE, Davies NB. Female cuckoo calls misdirect host defences towards the wrong enemy. Nat Ecol Evol. 2017;1:1520-5.

Yu J, Wang L, Xing X, Yang C, Ma J, Møller AP, Wang H, Liang W. Barn swallows (Hirundo rustica) differentiate between common cuckoo and sparrowhawk in China: alarm calls convey information on threat. Behav Ecol Sociobiol. 2016;70:171-8.

Yu J, Xing X, Jiang Y, Liang W, Wang H, Møller AP. Alarm call-based discrimination between common cuckoo and Eurasian sparrowhawk in a Chinese population of great tits. Ethology. 2017;123:542-50.

Zheng G. A checklist on the classification and distribution of the birds of China. 3rd ed. Beijing: Science Press; 2017.
Ready to submit your research? Choose BMC and benefit from:

- fast, convenient online submission

- thorough peer review by experienced researchers in your field

- rapid publication on acceptance

- support for research data, including large and complex data types

- gold Open Access which fosters wider collaboration and increased citations

- maximum visibility for your research: over $100 \mathrm{M}$ website views per year

At BMC, research is always in progress.

Learn more biomedcentral.com/submissions 